\title{
Estudio de patología funcional tiroidea en la población adulta de un centro de Atención Primaria
}

\author{
Mạ J. Sender Palacios, Mạ Vernet Vernet*, A. Franco Murillo**, Mạ A. \\ ORTEGa TALlón**, S. PÉrez LóPez***, M. FARO Colomés*** \\ Especialista en Medicina de Familia y Comunitaria. Doctora en Mediana y Cirugía. \\ *Especialista en Mediana Interna. Doctora en Mediana y Cirugía. **Espedalista en \\ Mediana de Familia y Comunitaria. ${ }^{* * *}$ Residente de M edicina de Familia y Comunita - \\ ria. Centro de Atención Primaria Ter rassa Nord. Unidad Docente de M edicina de Fami - \\ lia y Comunitaria del Consorci Sanitari de Terrassa. Barcelona
}

\section{RESUMEN}

Fundamentos: la patología tiroidea es un moti vo de consulta frecuente en Atención Primaria. El conocimiento de la situación de la misma en su en torno facilita su manejo.

Objetivo: conocer prevalencia y tipo de patolo gía funcional tiroidea en población adulta atendi da en un Centro de Atención Primaria.

Diseño: estudio descriptivo transversal.

Material y métodos: población mayor de 14 años adscrita a dos Unidades Básicas Asistencia les. Muestra de 442 personas, analizándose edad, sexo, determinación de tirotropina y patología ti roidea previa.

Resultados: personas incluidas: 375 [edad me dia $46 \pm 17$ años; $61,3 \%(n=230)$ mujeres]. Patología tiroidea previa: 16 casos (4\%). Patología funcional tiroidea activa: 14 casos $(3,63 \%)$ distribuidos entre 11 pacientes con aumento de tirotropina $(78,6 \%)$ y 3 pacientes con disminución de tirotropina $(21,4 \%)$. Prevalencia de patología funcional tiroidea: global del 5,9\% (22 casos, 12 de nuevo diagnóstico); $8,7 \%(n=20)$ en mujeres; $1,3 \%(n=2)$ en hombres; $5,9 \%(n=11)$ en el grupo menor a 50 años y 5,5\% $(n=11)$ en el grupo de 50 o más años. Tipos patología: 0,8\% (n=3) hipotiroi dismo clínico; $3,7 \%(n=14)$ hipotiroidismo subclí nico; $0,3 \%(n=1)$ hipertiroidismo clínico; $1 \%$ $(n=4)$ hipertiroidismo subclínico.

Conclusiones: tanto la prevalencia global de patología funcional tiroidea como la distribución de la misma por tipo de patología y sexo, no difie ren de las descritas en la literatura. Se observan
Functional thyroid disease in an adult population from an Primary Health Care center

\section{ABSTRACT}

Backgrounds: thyroid diseases are a common consultation in Primary Health Care. The know ledge of the situation in its area facilitates its ma nagement.

Objective: to know functional thyroid disease prevalence and type in an adult population atten ded from an Primary Health Care Center.

Design: transversal descriptive study.

Material and methods: sample of 442 people. Variables: age, sex, thyroid-stimulating hormone and previous thyroid disease.

Results: 375 people included [average age $46 \pm 17$ years; $61,3 \%(n=230)$ women]. Previous thyroid disease: 16 cases (4\%). Active functional thyroid disease: 14 cases $(3,63 \%)$ distributed in 11 patients with thyroid-stimulating hormone increa sed $(78,6 \%)$ and 3 patients with thyroid-stimu lating hormone deficient $(21,4 \%)$. Functional thyroid disease prevalence: total 5,9\% (22 cases, 12 of them undiagnosticated previously); 8,7\% $(n=20)$ in women; $1,3 \%(n=2)$ in men; $5,9 \%(n=11)$ in the under 50 years of age group and 5,5\% $(n=11)$ in the 50 years and more of age group. Kind of disease: $0,8 \%(n=3)$ clinical hypothyroidism; $3,7 \%(n=14)$ subclinical hypothyroidism; $0,3 \%(n=1)$ clinical hyperthyroidism and $1 \%(n=4)$ subclinical hy pertyroidism.

Conclusions: both functional thyroid disease to tal prevalence and distribution by kind of patho logy and sex are not different from described in other studies. We find some differences in relation to the age groups, with a less prevalence in elderly

Aceptación: 15-01-02 
diferencias respecto a la distribución por edades, con una menor prevalencia en edades avanzadas de la vida. El elevado porcentaje de nuevos diag nósticos, implica reflexionar sobre la necesidad de aumentar la sospecha clínica de la patología fun cional tiroidea.

Palabras clave: Patología tiroidea. Atención Primaria. Prevalencia. people. The high rate in new diagnosis make us to think about the need of increase the clinical sus pect of functional thyroid disease.

Key words: Thyroid disease. Primary Health Care. Prevalence.

\section{INTRODUCCIÓN}

La patología de la glándula tiroides representa, después de la diabetes mellitus tipo 2, el motivo de consulta endocrinológica más frecuentemente atendido por el médico de familia ${ }^{1}$. Dentro de la misma se incluyen diversos tipos de alteraciones, siendo las más frecuentes las relacionadas con las diferentes formas de patología funcional tiroidea $(\mathrm{PFT})^{2}$. La prueba de laboratorio inicial más adecuada para el diagnóstico de la disfunción tiroidea es la determinación de la hormona tirotropina hipofisaria $(\mathrm{TSH})^{3-7}$.

La prevalencia de PFT descrita en diferentes poblaciones, varía ampliamente dependiendo de diversos factores entre los cuales se pueden hallar los rangos de TSH según la técnica empleada para su determinación, la definición analítica de hipotiroidismo subclínico, las edades, la distribución poblacional y el contenido en yodo de las poblaciones estudiadas. Así, según diferentes estudios, dicha prevalencia puede oscilar desde una cifra inferior al $0,5 \%$ para el hipertiroidismo a un $16 \%$ para el hipotiroidismo subclínico en población de más de 60 años ${ }^{8-14}$.

Dada la considerable prevalencia de disfunción tiroidea en la población general y lo fundamental de su detección precoz con el fin de evitar la repercusión que la disfunción tiroidea produce a diferentes niveles del organismo (cardiovascular, cognitivo, metabolismo óseo) es importante el papel del médico de Atención Primaria a dicho nivel del diagnóstico.

El objetivo del presente estudio es el de conocer la prevalencia de Patología Funcional Tiroidea en la población adulta atendida en un Centro de Atención Primaria.

\section{MATERIAL Y MÉTODOS}

Estudio descriptivo transversal realizado en un Centro de Atención Primaria de ámbito urbano, de la ciudad de Terrassa, con una cobertura poblacional de 40.000 habitantes.

\section{Población a estudio}

-Criterios de inclusión: se incluyó una muestra representativa de toda la población mayor de 14 años adscrita a dos unidades básicas asistenciales.

- Criterios de exclusión: patología terminal crónica y negativa del paciente.

-Cálculo de la muestra: de las 4.504 personas que cumplían los criterios de inclusión, se consideró como representativa, a través de un muestreo aleatorio simple, una muestra de 442, asumiendo una proporción esperada del $1 \%$, una precisión del $1 \%$ y un intervalo de confianza del $95 \%$. Las pérdidas, incluidas en la muestra mencionada, se calcularon en un $20 \%$.

\section{Mediciones}

De cada uno de los sujetos incluidos en el estudio se analizaron las siguientes variables: a) demográficas: edad y sexo; b) clínicas: existencia de patología tiroidea previa documentada, tratamiento anterior y actual de la misma; y c) analíticas: determinación de tirotropina (TSH) y determinación de tiroxina libre (T4) en los sujetos con valores alterados de la TSH.

La TSH se realizó en todos los casos, con excepción de los que ya tenían una determinación de la misma en los tres meses previos al estudio, por existencia de patología tiroidea previa o por cualquier otro motivo.

Se englobaron dentro del término de Patología Tiroidea Funcional Activa todas las alteraciones de la TSH en el momento del estudio, consecuencia o de un nuevo diagnóstico o de un mal control de la patología tiroidea previamente conocida.

Según nuestro laboratorio de referencia, las cifras de normalidad de los parámetros analíticos mencionados fueron los siguientes: TSH de 0,25 a $6,70 \mu \mathrm{UI} / \mathrm{mL}$ y T4 de 9,00 a 24,00 $\mathrm{pmol} / \mathrm{L}$.

La citación de la población seleccionada se llevó a cabo a través de una carta informativa, realizándose la recaptación a través de una segunda carta. 
Para la identificación de la población a estudio se utilizó el registro informatizado del centro. Todas las variables se obtuvieron a través del interrogatorio directo al usuario y de la revisión activa de los datos existentes en sus correspondientes historias clínicas. El estudio analítico se llevó a cabo previo consentimiento informado de cada una de las personas incluidas en el estudio. El periodo de realización del mismo fue de enero a octubre de 2000 .

\section{Estudio estadístico}

El análisis estadístico descriptivo se presenta como media y desviación estándar para las variables cuantitativas y como porcentajes para las variables cualitativas. En el análisis bivariado se ha utilizado el análisis de la varianza de un factor para comparar variables cuantitativas y cualitativas, utilizándose la prueba no paramétrica de KruskalWallis en el caso de que el resultado de la homogenización lo exigiera. La prueba del Chi cuadrado se utilizó para la comparación de variables cualitativas. El nivel de significación estadística considerado fue del $5 \%$.

\section{RESULTADOS}

En total se estudiaron 375 personas, lo cual representa un $85 \%$ de la muestra seleccionada, con una edad media de $46 \pm 17$ años y cuya distribución por grupos de sexo y edad se muestran en la tabla I.

La media de TSH fue superior en las mujeres $(2,91 \pm 2,93 \mu \mathrm{UI} / \mathrm{mL}$; IC $2,53-3,29)$ respecto a la obtenida en los hombres $(2,21 \pm 1,48 \mu \mathrm{UI} / \mathrm{mL}$; IC $1,97-2,45)(p=0,008)$. No se observaron diferencias significativas respecto a la edad, siendo la media de TSH en el grupo de personas con menos de 50 años de $2,72 \pm 2,54 \mu \mathrm{UI} / \mathrm{mL}$ (IC 2,35-3,09) y en el de más de 50 años de $2,56 \pm 2,45 \mu \mathrm{UI} / \mathrm{mL}$ (IC $2,21-2,91)(\mathrm{p}=0,54)$.

\begin{tabular}{|c|c|c|}
\hline \multicolumn{3}{|c|}{$\begin{array}{c}\text { DISTRIBUCIÓN POR EDAD Y SEXO DE LA POBLACIÓN } \\
\text { A ESTUDIO }\end{array}$} \\
\hline Sexo/Edad & $\begin{array}{c}n \\
\text { (total 375) }\end{array}$ & $\%$ \\
\hline Hombres & 145 & 38,7 \\
\hline Mujeres & 230 & 61,3 \\
\hline 15-49 años & 185 & 49,3 \\
\hline$>49$ años & 190 & 50,7 \\
\hline
\end{tabular}

\section{Patología tiroidea previa}

Del total de la población estudiada, un $4 \%$ $(\mathrm{n}=16)$ tenían antecedentes de algún tipo de patología tiroidea. Todas eran mujeres con una edad me-

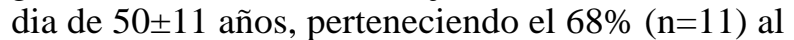
grupo de edad superior o igual a 50 años y el $32 \%$ $(n=5)$ al de menos de cincuenta. No se observaron diferencias significativas entre los porcentajes obtenidos para ambos grupos de edad (edad media de 50,5 $\pm 11,4$ años; IC 44,4-56,6 para el grupo de menos de 50 años y de $45,7 \pm 16,8$; IC 43,9-47,4 para el otro grupo).

Referente al tipo de patología previa se recogieron los diagnósticos descritos en la tabla II. Tampoco se observaron diferencias significativas, en cuanto a la edad, entre los diferentes tipos de patología tiroidea conocida.

\section{Patología tiroidea funcional activa}

El total de pacientes con PFT activa fue de 14, lo cual representaba una proporción del 3,6\%, agrupados entre 11 pacientes $(78,6 \%)$ con aumento de la TSH y 3 pacientes $(21,4 \%)$ con disminución de la TSH. En la tabla III se describe la distribución por sexo y edad de estos pacientes. La edad media de los pacientes con hipotiroidismo fue de 43,8 812,9 años (IC 35,1-52,4), mientras que la de los de hipertiroidismo fue de 38,6 $\pm 11,71$ años (IC 9,5-67,7) $(\mathrm{p}=0,68)$.

Del grupo de pacientes con aumento de la TSH, o hipotiroidismo, un $27 \%(n=3)$ tenían patología previa: uno con hipotiroidismo autoinmune en actual tratamiento sustitutivo con levotiroxina, uno con bocio intervenido quirúrgicamente y uno con tiroiditis de De Quervain. Dos de las pacientes con disminución de la TSH, o hipertiroidismo, tenían antecedentes de carcinoma de tiroides y realizaban tratamiento substitutivo post-quirúrgico,

\begin{tabular}{|lc|}
\hline \multicolumn{1}{|c}{ Tabla II } & \\
\hline TIPOS DE PATOLOGÍA TIROIDEA PREVIA & \\
\hline Patología tiroidea previa & $n$ \\
\hline Hipertiroidismo clínico & 1 \\
\hline Hipertiroidismo subclínico & 1 \\
\hline Hipotiroidismos clínicos & 2 \\
\hline Hipotiroidismos subclínicos & 5 \\
\hline Cacios normofuncionantes & 4 \\
\hline Tiroiditis de De Quervain & 2 \\
\hline
\end{tabular}




\begin{tabular}{|c|c|c|}
\hline \multicolumn{3}{|c|}{$\begin{array}{c}\text { DISTRIBUCIÓN POR EDAD Y SEXO DE LA PATOLOGÍA } \\
\text { FUNCIONAL TIROIDEA ACTIVA }\end{array}$} \\
\hline Sexo/Edad & $\begin{array}{c}\text { Aumento TSH } \\
n \% \\
\text { (total 11) }\end{array}$ & $\begin{array}{c}\text { Disminución TSH } \\
n \% \\
\text { (total 3) }\end{array}$ \\
\hline Hombres & $2(18,2 \%)$ & $0(0 \%)$ \\
\hline Mujeres & $9(81,8 \%)$ & $3(100 \%)$ \\
\hline 15-49 años & $5(45,4 \%)$ & $2(66,6 \%)$ \\
\hline$>49$ años & $6(54,6 \%)$ & $1(33,3 \%)$ \\
\hline
\end{tabular}

manteniendo un hipertiroidismo subclínico farmacológico.

\section{Prevalencia de la PFT}

La prevalencia global de PFT fue del 5,9\% ( $\mathrm{n}=22$ casos), siendo 12 de ellos $(54,5 \%)$ de nuevo diagnóstico (Fig. 1). La prevalencia de PFT obtenida por sexos y la distribuida por edades se muestra en la tabla IV. En lo referente a la edad, no existieron diferencias entre el grupo de pacientes de nuevo

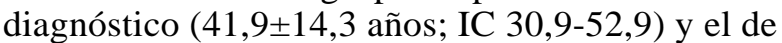
diagnóstico conocido $(44,2 \pm 9,3$; IC 32,6-55,8) $(\mathrm{p}=0,75)$.

Respecto al tipo de PFT, el diagnóstico más frecuente fue el del hipotiroidismo subclínico con 14 casos, seguido del hipertiroidismo subclínico con 4, del hipotiroidismo clínico con 3 y del hipertiroidismo clínico con 1. La prevalencia de dicha P.F.T.

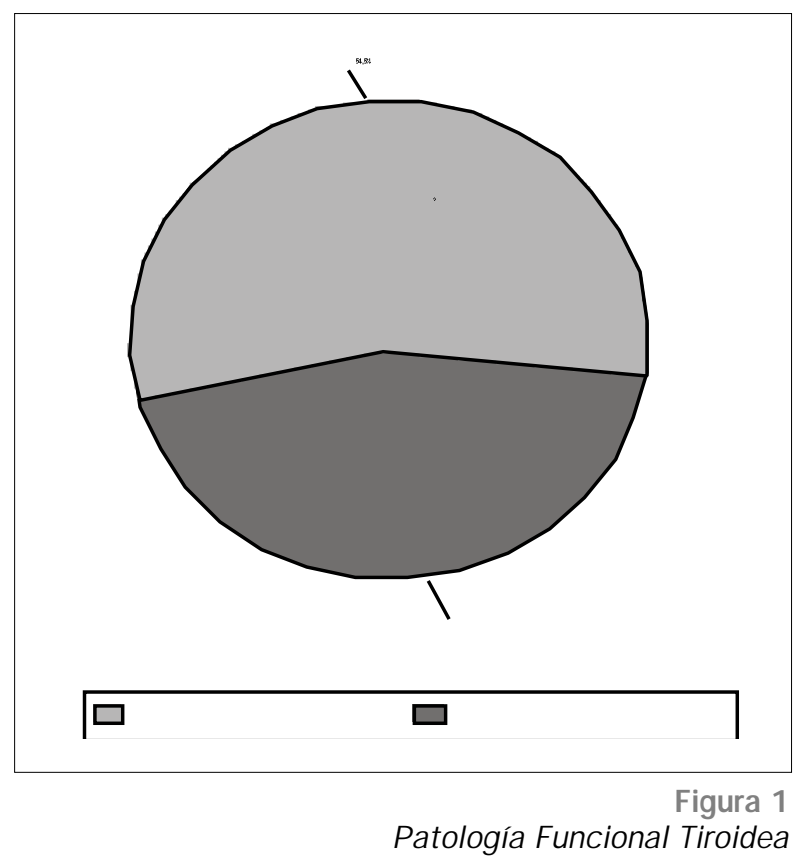

\begin{tabular}{|c|c|c|}
\hline \multicolumn{3}{|c|}{$\begin{array}{l}\text { DISTRIBUCIÓN POR EDAD Y SEXO DE LA PREVALENCIA DE LA } \\
\text { PATOLOGÍA FUNCIONAL TIROIDEA }\end{array}$} \\
\hline Sexo/Edad & $\begin{array}{c}n \% \\
\text { (total 11) }\end{array}$ & $\% *$ \\
\hline $\begin{array}{l}\text { Hombres } \\
\text { Mujeres }\end{array}$ & $\begin{array}{c}2 \\
20\end{array}$ & $\begin{array}{l}1,4 \\
8,7\end{array}$ \\
\hline $\begin{array}{l}\text { 15-49 años } \\
>49 \text { años }\end{array}$ & $\begin{array}{l}11 \\
11\end{array}$ & $\begin{array}{l}5,9 \\
5,8\end{array}$ \\
\hline
\end{tabular}

respecto a los tipos de la misma, se muestra en la figura 2 .

En las mujeres, la prevalencia de hipotiroidismo subclínico fue del 5\% $(n=12)$, de hipertiroidismo subclínico del 1,7\% (n=4), de hipotiroidismo clínico del $1,3 \%(n=3)$ y de hipertiroidismo clínico del $0,4 \%(n=1)$. Los dos únicos casos de PFT en hombres correspondieron a hipotiroidismo subclínico (prevalencia 1,4\%).

No se objetivaron diferencias en la edad respecto a los diferentes tipos de patología. Así, la edad media de los pacientes con hipotiroidismo fue de $48 \pm 13,5$ años (IC 41,03-54,96), la de los pacientes con hipotiroidismo subclínico fue de 48,71 $\pm 14,4$ años (IC 40,3-57,06) mientras que la de los pacientes con hipertiroidismo fue de 46,2 $\pm 14,8$ años $(27,7-64,62)(p=0,96)$.

\section{DISCUSIÓN}

El papel del médico de Atención Primaria en el manejo de la patología tiroidea es importante, tanto por la considerable prevalencia de ésta, como por requerir un alto grado de sospecha clínica por la asociación con otras patologías también muy prevalentes dentro del ámbito de la Atención Primaria, sobre todo en el caso del hipotiroidismo ${ }^{11,12}$. El diagnóstico,

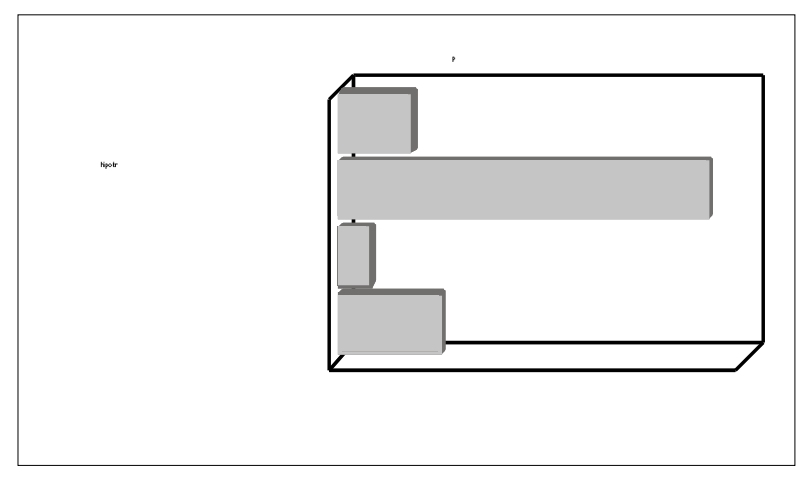

Figura 2

Prevalencia según el tipo de Patología Funcional Tiroidea 
como mínimo de sospecha, de la patología funcional tiroidea desde el ámbito de la Atención Primaria es fácilmente asumible, pudiendo ser detectada mediante la determinación de $\mathrm{TSH}^{4,12}$. Además, el médico de familia puede desarrollar un papel importante tanto en el tratamiento y seguimiento de algunas de las alteraciones de la glándula tiroides como en la correcta derivación del paciente a la Atención Especializada, con el fin de mejorar los resultados de algún estudio publicado donde la derivación está justificada solamente en la mitad de los casos ${ }^{1}$.

Para asumir de forma más correcta una determinada patología, es importante conocer la situación de la misma en cada entorno. En este sentido la prevalencia conocida de la patología tiroidea varía dependiendo de múltiples factores según los diferentes estudios realizados, oscilando las cifras, en nuestro país, entre un $0,6 \%$ y un $2,74 \%{ }^{2,8}$. Los resultados de este trabajo muestran una prevalencia discretamente superior a las mencionadas.

Referente a la PFT, la prevalencia descrita en diferentes poblaciones, varía ampliamente dependiendo de diversos factores como pueden ser diferentes rangos de TSH, diferentes definiciones analíticas de hipotiroidismo subclínico, diferentes edades, distribución poblacional y diferente contenido en yodo de las poblaciones estudiadas ${ }^{8,9,12-14}$. Así, los valores de prevalencia de hipotiroidismo subclínico oscilan entre un 2 y un $15 \%$, con una incidencia global entre un 2 y un $5 \%$ de casos nuevos por año ${ }^{14}$. Entre la población afecta de hipotiroidismo subclínico, la incidencia de hipotiroidismo clínico puede oscilar entre un 5 y un $15 \%$ cada año ${ }^{13}$. La prevalencia de hipertiroidismo es menor en la mayoría de los trabajos, aunque se han referenciado cifras globales para el hipertiroidismo subclínico que oscilan entre un 1,5 y un $12 \%$ con una incidencia a hipertiroidismo clínico del 1,5\% por año y del $2 \%$ a los 10 años ${ }^{14}$.

En nuestro país, la mayoría de los trabajos realizados han sido sobre población con patología tiroidea conocida, sin embargo en el estudio llevado a cabo en la provincia de Lleida, en población general, se obtuvieron prevalencias de hipotiroidismo del $3,48 \%$ y de hipertiroidismo del $5,62 \%$, con un 2,23 y un $4,6 \%$ de subclínicos respectivamente 9 . El presente estudio, llevado a cabo en población atendida pero no demandante, muestra respecto al estudio de Gascó ${ }^{9}$ cifras similares de prevalencia de hipotiroidismo, siendo inferiores las de hipertiroidismo, sin embargo estas últimas difieren poco de las descritas en el global de la literatura. El hecho de que la población estudiada sea población atendida, es decir, población con historía clínica abierta en el centro, no representaría un sesgo del estudio, ya que dado el funcionamiento de dicho centro desde hace más de diez años la cobertura poblacional se considera del $100 \%$.
Respecto al sexo, este estudio muestra una gran predominio de la patología tiroidea en el sexo femenino, lo cual concuerda con todos los trabajos publicados, mostrando muchos de ellos proporciones superiores al $80 \%$ de mujeres, sobre todo en los casos de hipotiroidismo ${ }^{8,9,13-15,16-18}$. Según el trabajo de Rodríguez Carrillo ${ }^{19}$, el médico de Atención Primaria solicita con mucha mayor frecuencia pruebas de función tiroidea a las mujeres.

Referente a la edad, las referencias destacan que es una patología con mayor prevalencia entre la $3^{\mathrm{a}}$ y $6^{\mathrm{a}}$ década de la vida ${ }^{12}$, aumentando la misma en edades más avanzadas con valores referidos de hasta un $16 \%{ }^{11,14}$. En estudios realizados en poblaciones de más de 55 años, se han descrito prevalencias para el hipertiroidismo del $2 \%$ y para el hipotiroidismo entre el 4,4 y el $15 \%$, incluyendo los casos de subclínico ${ }^{15-17}$, sin diferencias significativas, respecto a la edad, a partir de los 60 años $^{18}$. En estudios realizados en nuestro entorno, la edad media de la población con patología tiroidea oscila entre 47 y 57 años ${ }^{8,9}$. Según el estudio de Rodríguez Carrillo ${ }^{19}$ la edad media de solicitud de las pruebas de función tiroidea es de 44 años, observando, al igual que refieren otros trabajos, que dicha exploración se realiza poco en personas de edad avanzada. En este trabajo, la mayor frecuencia de patología tiroidea, tanto conocida como de nuevo diagnóstico, se halla entre los 40 y 60 años, siendo la prevalencia de PFT similar entre los dos grupos de edad valorados. Quizás hubiera cabido esperar que los pacientes con patología conocida pertenecieran al grupo de mayor edad por ser los que con más frecuencia acuden al centro de salud y a los que se les realiza más exploraciones complementarias. La media de edad de PFT tanto activa como total es similar a la descrita por Gascó ${ }^{9}$ para el hipotiroidismo e inferior para el hipertiroidismo. La baja prevalencia obtenida en este trabajo en población de edad más avanzada, podría estar en relación con las características de la muestra a estudio, que se obtuvo a través de muestreo aleatorio de toda la población adulta, resultando una mayor proporción de personas con edades comprendidas entre 40 y 60 años. Posiblemente un estudio dirigido al grupo de personas de mayor edad podría aumentar la prevalencia de la patología estudiada en ellos, sin embargo, creemos que las cifras en nuestros ancianos no llegarían a ser tan altas como las descritas en otros estudios. A pesar de que en este estudio no se valoraron las características de la población considerada como pérdidas, el porcentaje de mujeres $(61,3 \%)$ y de personas de 50 o más años $(50,7 \%)$ estudiadas resultó ser superior al porcentaje existente, de estos grupos, en la población registrada $(55 \%$ mujeres y $35 \%$ de 50 años o más). Al ser estos dos colectivos los 
que presentan habitualmente una mayor prevalencia de patología tiroidea, se considera que las pérdidas no influyeron en los resultados obtenidos.

El cribado sistemático de la patología tiroidea probablemente no está indicado en toda la población adulta, sin embargo según diversas asociaciones y grupos de expertos las recomendaciones, ya sea en publicaciones o guías de práctica clínica, son diversas ${ }^{20}$. Algunas lo recomiendan en poblaciones donde la prevalencia e incidencia de esta patología es más elevada (mujeres de más de 50 años según la American Collegge of Physicians-Ameri can Society of Internal Medicine $\left.{ }^{6}\right)$, otras hacen una recomendación más amplia (cada 5 años a partir de los 35 años en toda la población según el American Thyroid Association ${ }^{21}$ ), mientras que otras no recomiendan realizar ningún tipo de cribado en población no sintomática (United States Preventive Ser vices Task For C $^{20}$ ). En nuestro ámbito, las recomendaciones van dirigidas a realizar pruebas tiroideas a pacientes con elevado riesgo de padecer alteraciones hormonales, ya sea por sospecha clínica o por presencia de factores de riesgo (bocio, hipercolesterolemia, anemia refractaria, taquiarritmias, uso de determinados fármacos: amiodarona, litio $)^{7}$. Por su parte, el Programa de Actividades Preventivas y Promoción de la Salud (PAPPS) de la Sociedad Española de Medicina de Familia y Comunitaria, recomienda el estudio, para detectar el hipotiroidismo, a partir de los 50 años si existe algún síntoma general atribuible a enfermedad tiroidea $^{22}$.

De los resultados del presente estudio se puede concluir que tanto la prevalencia global de la PFT como la distribución de la misma por tipo de patología y sexo, no difieren de las descritas en la literatura. Sin embargo sí se observan diferencias respecto en la distribución por edades, con una menor prevalencia en edades avanzadas de la vida. Así mismo, y a pesar de la considerable prevalencia de patología tiroidea previa, el hecho de que más de la mitad de los casos no estuvieran diagnosticados, hace reflexionar sobre la necesidad de aumentar la sospecha clínica en la detección de la PFT, con especial atención en los casos con poca o nula sintomatología, sobre todo si estos pacientes pertenecen a grupos de riesgo y a población de mayor edad con el fin de evitar un infradiagnóstico de la mencionada patología.

CORRESPONDENCIA:

$M^{\mathrm{a}}$. J. Sender Palacios

CAP Terrassa Nord

C/ 25 de Setembre, 26

08226 Terrassa. Barcelona

Tel.: 937310268 - Fax: 937313080

e-mail: terrnord@galenics.com

\section{Bibliografía}

1. Fuertes Goñi C, Loayssa Lara JR, Pérez Etxarri JM, Urrutia Sanzberro J, Artetxe Andrés A, Menéndez Torre E. Derivación a endocrinología por patología tiroidea. Calidad y justificación. Aten Primaria 2001; 27: 489-96.

2. Álvarez Nido R, Pérez Calpena C, Alonso Roca, Monereo Mejías S. Papel del médico de atención primaria en el diagnóstico y seguimiento de la patología tiroidea. Aten Primaria 1994; 14: 877-9.

3. Polo Polo MJ, Ricós Aguilá C, Catalán Gili R, Juvany Roig R. Aplicación clínica de los datos de variabilidad biológica de las hormonas tiroideas. Med Clin (Barc) 1992; 99: 529-31.

4. Riesco Prieto M, Barceló Bennassar A, Pérez Esteban G, Ribes Vallés JL. Valoración de un protocolo diagnóstico de disfunción tiroidea. Aten Primaria 1997; 20: 355-60.

5. Surks M, Chopra I, Mariash C, Nicoloff J, Solomon D. American Thyroid Association Guidelines for Use of Laboratory Tests in Thyroid Disorders. JAMA 1990; 263: 1529-32.

6. American College of Physicians. Clinical Guideline, Part 1. Screening for Thyroid Disease. Ann Intern Med 1998; 129:141-3.

7. Álvarez Vicente JC. Pruebas funcionales tiroideas. On line: www.fisterra.com/guias2/tiroideas.htm

8. Serra, Méndez MA, Davins J, Borrell M, Baxarias J, Ríos L. Patología tiroidea en un centro de salud. Aten Primaria 1995; 15: 457-60.
9. Gascó Eguiluz E, Serna Arnaiz MC, Vázquez Torguet A, Peremiquel Lluch M, Ibarz Excuer M, Serra Majem L. Prevalencia de transtorno de la función tiroidea en la provincia de Lleida. Aten Primaria 1999; 24: 475-9.

10. Serna Arnaiz MC, Serra i Majem L, Gascó Eguiluz E, Muñoz Márquez J, Ribas L, Escobar del Rey F. Situación actual de la endemia de bocio y del consumo de yodo en la población del Pirineo y de la comarca del Segrià de Lleida. Aten Primaria 1998; 22: 642-8.

11. Díaz Belinchón O, Alonso Val A. Hipotiroidismo subclínico: aproximación diagnóstico-terapéutica en atención primaria. Aten Primaria 1996; 18: 564-6.

12. García De Francisco $S$, Taboada Taboada $M$, Álvarez Vega P. Disfunción tiroidea subclínica. Medifam 2001; 11: 253-64.

13. Wiersinga WM. Subclinical hypothyroidism and hyperthyroidism. I. Prevalence and clinical relevance. Neth J Med 1995; 46: 197-204.

14. Torroba Arlegui I, Puigdevall Gallego V, Laudo Pardos C, San Martín Barrio L. Disfunción tiroidea subclínica en atención primaria. Aten Primaria 1998; 21: 245-50.

15. Bagchi N, Brown TR, Parish RF. Thyroid dysfunction in adults over age 55 years. A study in a urban US community. Arch Inter Med 1990; 150: 785-7. 
16. Bemben DA, Winn P, Hamm RM, Morgan L, Davis A, Barton E. Thyroid disease in the elderly. Part 1. Prevalence of undiagnosed hypothyroidism. J Fam Pract 1994; 38: 577-82.

17. Sawin CT, Castelli WP, Hersshman JM, McNamara P, Bacharach $\mathrm{P}$. The aging thyroid. Thyroid deficiency in the Framingham Study. Arch Intern Med 1985; 145: 1386-8.

18. Sawin CT, Geller A, Hershman JM, Castelli W, Bacharach $\mathrm{P}$. The aging thyroid. The use of thyroid hormone in older persons. JAMA 1989; 261: 2653-5.

19. Rodríguez Carrillo C, Minué Lorenzo C, Bru Amategui S, Calvo Corbella C. Estudio descriptivo del uso de pruebas de función tiroidea (PFT) en un centro de salud. Aten Primaria 1995; 16: 57-8.

20. National Guidelines Clearinghous TM (NGC TM) Guidelines Synthesis. Screening for Thyroid Disease. On line: www.guideline.gov/COMPARISONS/THYROID1.asp

21. Ladenson PW, Singer PA, Ain KB, Bagchi N, Bigos ST, Levy EG, et al. American Thyroid Association Guidelines for Detection of Thyroid Dysfunction. Arch Intern Med 2000; 160: 1573-5.

22. Programa de Actividades Preventivas y Promoción de la Salud (PAPPS). Sociedad Española de Medicina de Familia y Comunitaria. On line: www.papps.org/menu22.htm 